\section{Where Experts Meet to Exchange Knowledge: Biotechnet's Summer School on Advanced Biotechnology}

Elsbeth Heinzelmann, Journalist science + technology

Abstract: Science and progress thrive on an open mind and a transnational exchange of ideas and experience. That's why, in 2005, two scientists with a vision launched the biotechnet Summer School on Advanced Biotechnology. This year, too, the Summer School - now held at ZHAW in Waedenswil - is attracting researchers from all over the globe, and is also celebrating its $10^{\text {th }}$ anniversary.

Keywords: Antibiotic resistance - Bacterial sythesis of metal chelators · Protein kinases in cancer therapy - Functionalized nanoparticles in release mechanism - Point-of-Care Therapeutic Drug Monitoring devices · Drug quantification in human blood

It all started out in 2005 when Dr. Daniel Gygax, President of biotechnet and Professor at the School of Life Sciences at FHNW Basel, went with Rolf Zaugg, an expert in further training, to the University of Palermo on the invitation of Professor Letizia Vittorelli, Biologa Cellulare e dello Sviluppo. "We wanted to foster a cooperation based upon three founding pillars, namely an exchange of students within the Erasmus programme, the organisation of an international Summer School on Advanced Biotechnology, and collaborative research on the basis of master and doctoral theses and postgraduate internships", Daniel Gygax recalls. The joint talks were fruitful and he discussed the matter with the biotechnet members at their next meeting. Everybody was in agreement upon the opportunity provided by such an event:

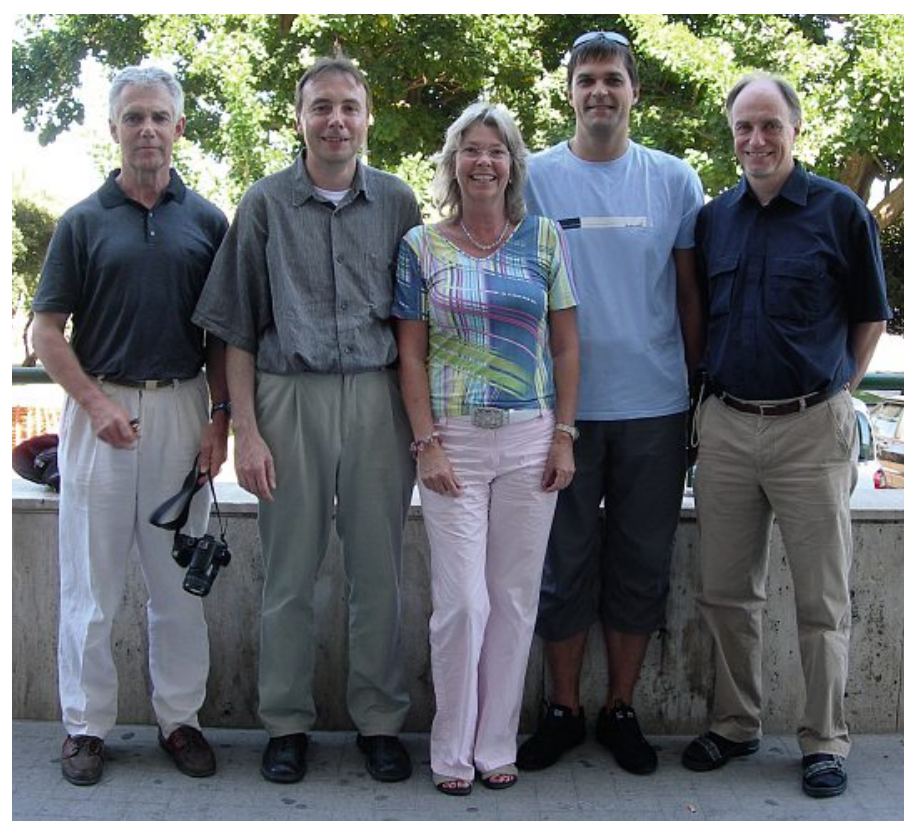

It all began in 2006 with the first Summer School at the University of Palermo and a small troop of biotechnet board members. Photo by ZHAW Wädenswil.

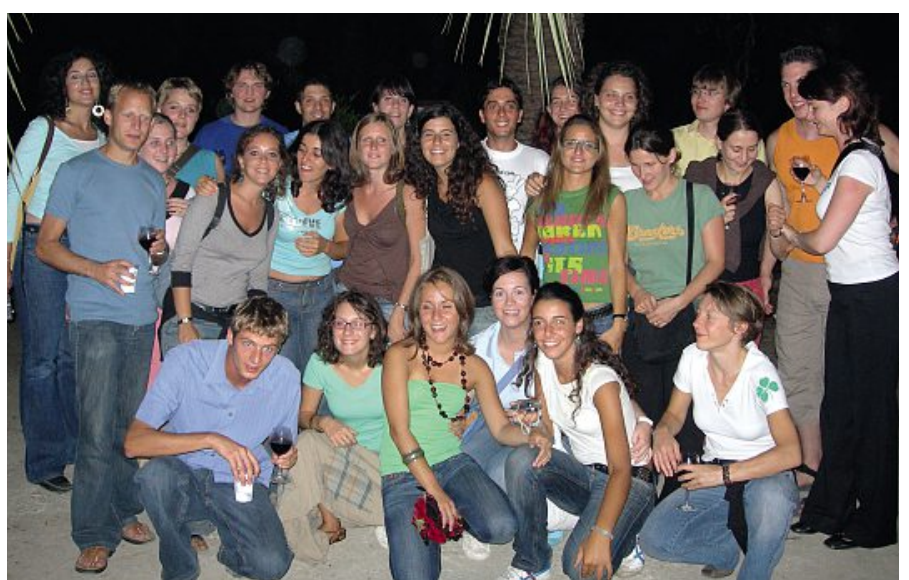

The students in 2006 showed huge interest in this professional gettogether: a source of motivation and an obligation for the initiators to continue this successful event. Photo by ZHAW Wädenswil.

members could network their locations with a joint approach and show students how to break down the barriers and give them a platform to present their work in an international setting. And so the International Summer School on Advanced Biotechnology was born.

\section{Knowledge is the only Resource which Multiplies when Shared}

The first Summer School took place at the University of Palermo in 2006, with lectures given mainly by the tutors. "We learned a lot about each other and brought together complementary disciplines from Palermo and Switzerland, which very quickly created synergies within biotechnet", comments Daniel Gygax.

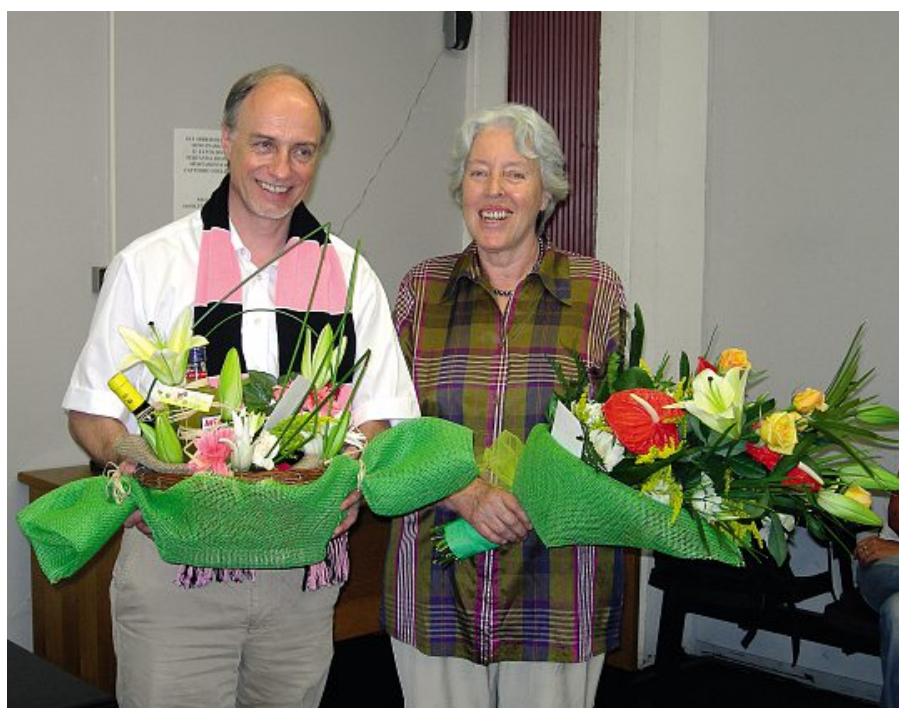

A very special thank-you from the students of this first course in 2006 to Professor Letizia Vittorelli at the University of Palermo and Professor Daniel Gygax, President of biotechnet. Photo by ZHAW Wädenswil. 
"As a visible sign of this development, students came from Palermo to Switzerland - first to FHNW Basel, and later also to HES-SO Sion to write their Master's theses."

The openness demonstrated sustainable success, as recalls Ursula Graf-Hausner, professor and research manager in cell culture technology and tissue engineering at ZHAW Waedenswil. "In 2006, Professor Italia di Liegro - who I got to know at the University of Palermo - called me to ask whether I needed a collaborator with expertise in cell biology. Indeed, I had new projects on the go and needed support. Soon Dr. Epifania Bono from Palermo began to work with us. Today, she speaks fluent German and, with her professional know-how, is indispensable in our team." Meanwhile, her husband has joined her at ZHAW Waedenswil and they have a young daughter.

\section{Exchange Knowledge so it Can Grow}

In 2015, the biotechnet Summer School celebrated its $10^{\text {th }}$ anniversary and took place at ZHAW Waedenswil, with international speakers. Professor Markus Seeger, Research Group Leader at the Institute for Medical Microbiology Zurich opened the meeting with Antibiotics, presenting antibiotic resistance mediated by drug efflux pumps. Antimicrobial resistance of human pathogenic bacteria is an emerging problem for global public health. It is often associated with the overproduction of membrane transport proteins capable to pump chemotherapeutics, antibiotics, detergents, dyes and organic solvents out of the cell. In Gram-negative bacteria such as Escherichia coli, tripartite multidrug efflux systems extrude a large variety of cytotoxic substances from the cell membrane directly into the medium bypassing the periplasm and the outer membrane. The trimeric outer membrane factor (OMF) TolC forms a $\beta$-barrel pore in the outer membrane and exhibits a long periplasmic $\beta$-helical conduit. The periplasmic membrane fusion protein (MFP) AcrA serves as a linker between TolC and the trimeric resistance nodulation cell division (RND) pump AcrB, located in the inner membrane acting as a proton/drug antiporter. The newly elucidated asymmetric structure of trimeric AcrB reveals three different monomer conformations representing consecutive states in a transport cycle. The monomers show tunnels with occlusions at different sites leading from the lateral side through the periplasmic porter (pore) domains towards the funnel of the trimer and TolC. The structural changes create a hydrophobic pocket in one monomer, which is not present in the other two monomers. Minocyclin and doxorubicin, both AcrB substrates, specifically bind to this pocket substantiating its role as drug binding pocket. The energy transduction from the proton motive force into drug efflux includes proton binding in (and release from) the transmembrane part. This novel transport model merges Jardetzky's alternate access pump mechanism with the rotating site catalysis of F1Fo ATPase and suggests a working hypothesis for the transport mechanism of RND transporters in general.

Contact: m.seeger@imm.uzh.ch

\section{What's New in Advanced Biotechnology}

In the domain of Bioanalytics, drug quantification in small amounts of blood is a topic of high interest in order to enable the development of novel Point-of-Care (POC) Therapeutic Drug Monitoring (TDM) devices. TDM supports the personalised treatment of patients by providing an optimal dose adjustment for therapeutics targeting diseases such as cancer or in organ transplantation cases. Currently, TDM is slow and costly, as it is performed in central analytical laboratories, and requires $\mathrm{ml}$ amounts of blood. In order to improve the situation for patients and tackle this inefficient process, the ISyPeM II project, funded by Nano-Tera.ch and the Swiss Confederation, aims to develop a compact and cost-effective POC device for TDM.

In the scope of this project, Diana Burghelea, a PhD student in the Diagnostic Systems Research Unit at the Institute of Life Technologies in Sion, is currently working on designing novel miniaturised immunoassays for drug quantification in human blood. The assays, which include key steps such as whole blood pre-treatment, mixing and reaction, are then incorporated into microstructures as a central element of the future POC TDM device.

Model of the prototype device (adapted from C. Guiducci, EPFL): One drop of whole blood is processed by a microfluidic disposable cartridge. The analytical setup measures the drug concentration and the result is then transferred to a database.

Contact: diana.burghelea@hevs.ch

Professor Wolfgang Wohlleben, Head of the Department for Microbiology/Biotechnology from the University of Tuebingen, is a pioneer in Biomaterials, especially the bacterial synthesis of metal chelators.

Chelating agents play a crucial role in the supply of microorganisms with trace. In addition to siderophores synthesised under iron starvation, the gram-positive soil bacterium Amycolatopsis japonicum produces the zincophore [S,S]-ethylene diamine-disuccinic acid (EDDS). EDDS is of industrial interest because it has similar properties to ethylenediaminetetraacetate (EDTA) which is widely used in the industry. Due to its poor degradability, EDTA became an environmental hazard. In contrast to EDTA, EDDS is biodegradable. Wolfgang Wohlleben and his team have elucidated EDDS biosynthesis and its regulation. The results are now used to establish a biotechnological production process for EDDS.

Contact: wolfgang.wohlleben@biotech.uni-tuebingen.de

\section{Network Promising Biotech Research}

In Cell Biology, the focus of Dr. Hugo Albrecht, Senior Lecturer in Pharmaceutical Science at the University of South Australia (UniSA), lies on protein kinases as targets for cancer therapy. Cancer is a leading cause of death worldwide, accounting for more than 7.6 million deaths every year. Several kinases have been validated as potential anti-cancer targets using cell biology and animal models. Hugo Albrecht and his colleagues at UniSA have developed a strategy to realise serine/threonine kinase inhibitors which specifically disrupt cell cycle progression, transcription and translation to improve future treatment of cancer.

Contact: hugo.albrecht@unisa.edu.au

In Applied Biotechnology, the primary interest of Giulio Ghersi, Professore Associato at Science e Technologie Biologiche Chimiche e Farmaceutiche at the University of Palermo, lies on his special drug delivery strategy, using functionalised nanoparticles in a targeting and release mechanism. Traditional chemotherapeutic applications, using molecules such as doxorubicin (Dox) and/or many others, have side effects due to an unspecific action. In order to obtain a specific release of drugs, Giulio Ghersi's team synthesised/built and conjugated different manufacturing nanoparticles (NPs) to specific drugs through a specific release system, such as a $\mathrm{pH}$-sensitive linker. Nevertheless, another problem to be solved was the specificity in the targeting. To do that, Ghersi and his colleagues developed different approaches by surface functionalisation using specific molecules having selective capability in cell targeting.

Contact: giulio.ghersi@unipa.it 


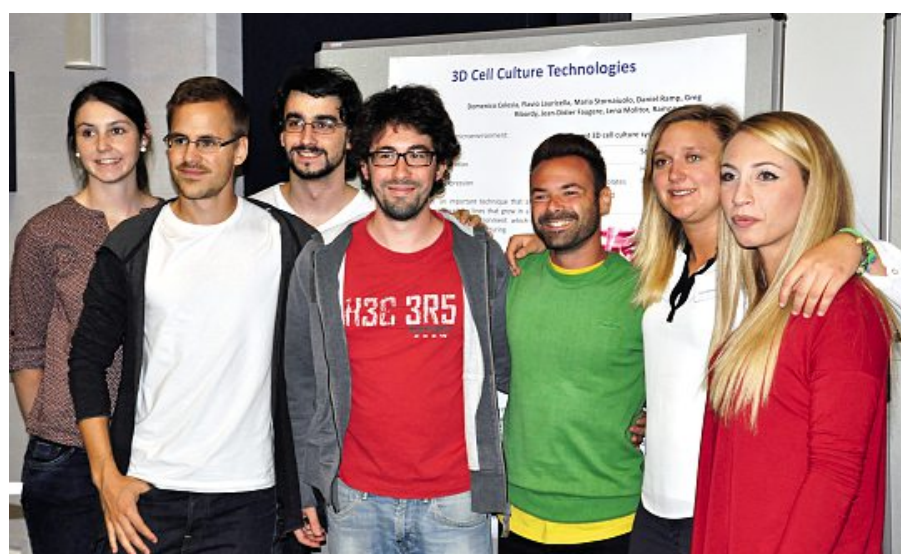

Students of the 2015 Summer School at the poster show in the auditorium at Waedenswil. They had to build internationally mixed groups to realise posters on specific topics in order to practice their cooperation skills. Photo by ZHAW Wädenswil.

Nowadays, the biotechnet Summer School takes place every year at a different research institute within Europe. After the retirement of Prof. Letizia Vittorelli, Prof. Anna Maria Puglia now organizes the biotechnet Summer School on behalf of the University of Palermo. And thanks to Daniel Gygax's excellent contacts to the University of Palermo and competence centres such as Management Center Innsbruck, it is still possible to bring together renowned experts from the biotech branch and to share scientific ideas and create cross-disciplinary links. Today the biotechnet Summer School for Advanced Biotechnology is a model of success.

www.biotechnet.ch

\section{Are you interested in meeting biotech experts?}

The 2016 Summer School will take place in Palermo at the end of August/beginning of September. For further information please contact:

Prof. Franz Baumberger, Organising Committee

E-mail: franz.baumberger@ biotechnet.ch

Tel.: 41766660175

Prof. Daniel Gygax, President of biotechnet Switzerland

E-mail: daniel.gygax@fhnw.ch

Tel.: 41614674562

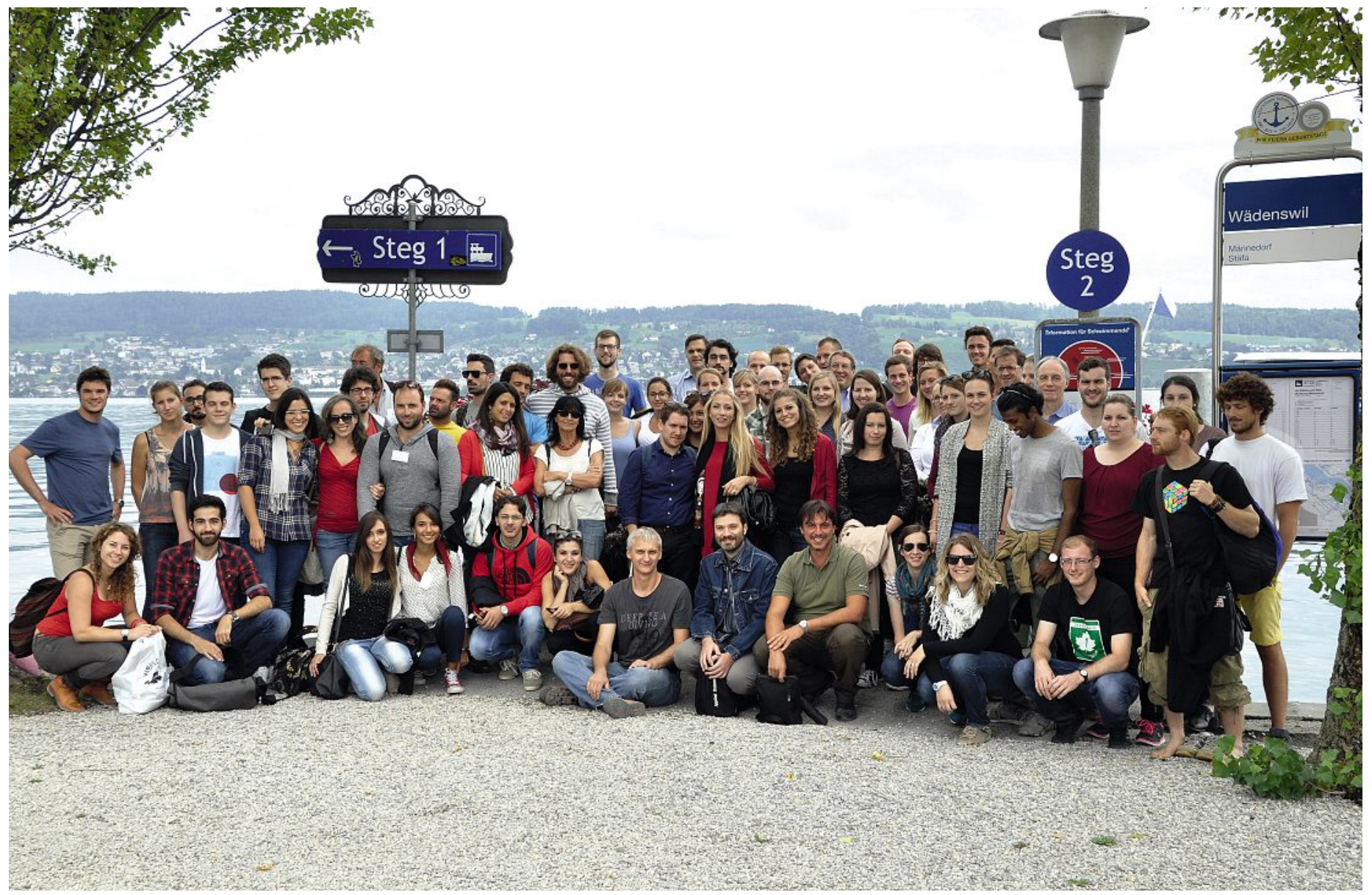

All participants of the 2015 Summer School meet at the pier in Waedenswil and wait for the scheduled cruise ship to depart to Zurich. Photo by ZHAW Wädenswil. 